\title{
Elevated expression of POLD1 is associated with poor prognosis in breast cancer
}

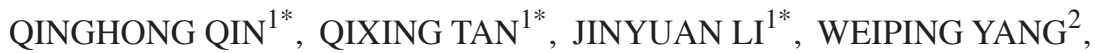 \\ BIN LIAN $^{1}$, QINGUO MO ${ }^{1}$ and CHANGYUAN WEI ${ }^{1}$ \\ Departments of ${ }^{1}$ Breast Surgery and ${ }^{2}$ Ultrasound Diagnosis, The Affiliated Tumor Hospital of \\ Guangxi Medical University, Nanning, Guangxi Zhuang Autonomous Region 530021, P.R. China
}

Received September 14, 2017; Accepted April 16, 2018

DOI: $10.3892 / \mathrm{ol} .2018 .9392$

\begin{abstract}
Polymerase $\delta$ catalytic subunit gene 1 (POLD1) may serve an important function in the development of tumors. However, its role in breast cancer remains unclear. The aim of the present study was to observe the expression and the function of POLD1 in breast cancer. A total of 84 patients with invasive breast carcinoma were recruited between 2011 and 2013. The expression of POLD1 was detected in paired tumor and adjacent normal tissues. Gene expression level of POLD1 was assessed using reverse transcription quantitative polymerase chain reaction. The protein expression of POLD1 was assessed using western blot analysis. The association between the clinicopathological features of patients with breast cancer and POLD1 expression was analyzed using a $\chi^{2}$ test. Disease-free survival (DFS) was analyzed using Kaplan-Meier method, and Cox regression analysis was performed to investigate clinicopathological significance of POLD1 expression. Additionally, the effects of POLD1 in regulating cell cycle and proliferation of MCF-7 cells were evaluated in vitro. The results demonstrated that gene and protein expression levels of POLD1 were significantly elevated in breast cancer tissues compared with those in adjacent normal tissues. Increased expression of POLD1 was significantly associated with positive lymph node status $(\mathrm{P}=0.028)$, histological grade $(\mathrm{P}=0.025)$, p53 status $(\mathrm{P}<0.001)$ and ki-67 index $(\mathrm{P}=0.020)$. Survival analysis demonstrated that increased expression of POLD1 was associated with poor DFS $(\mathrm{P}=0.033)$. Additionally, increased expression of POLD1 was associated with shorter DFS at early-stage $(\mathrm{P}=0.037)$, late-stage cases $(\mathrm{P}=0.023)$ and
\end{abstract}

Correspondence to: Professor Changyuan Wei, Department of Breast Surgery, The Affiliated Tumor Hospital of Guangxi Medical University, 71 Hedi Road, Nanning, Guangxi Zhuang Autonomous Region 530021, P.R. China

E-mail: gxzlrx@hotmail.com

*Contributed equally

Key words: breast carcinoma, polymerase $\delta$ catalytic subunit gene 1, prognosis, biomarker with the presence of triple-negative tumors (TNBC; $\mathrm{P}=0.049$ ). Multivariate analysis revealed that POLD1 may be used as an independent prognostic factor in patients with breast cancer. In vitro studies revealed that downregulation of POLD1 suppressed cell cycle progression and proliferation in MCF-7 cells. In conclusion, POLD1 may be considered as a potential prognostic marker for invasive breast carcinoma.

\section{Introduction}

Breast cancer is the most common cancer in women. Recurrence rate and mortality remains high in women with breast cancer (1). Despite advances, the clinical prognosis for breast cancer depends on the stage of the tumor. Further studies investigating the molecular mechanisms underlying the pathogenesis of breast cancer and identifying novel therapeutic approaches are required.

DNA polymerase is required for DNA replication in eukaryotic cells. DNA polymerase ( $\mathrm{Pol}) \delta$ belongs to the B family of DNA polymerases. Pol $\delta$ is the most important replicase in eukaryotic DNA replication and serves a primary role for the synthesis of lagging strand, and has a 3'-5'exonuclease activity. It also participates in DNA damage repair. Pol $\delta$ is involved in DNA replication and repair of damaged DNA, and mutations in Pol $\delta$ may be associated with the progression of cancer (2). DNA Pol $\delta$ consists of four subunits (p125, p68, p50 and $\mathrm{p} 12)(2)$. The 125 subunit is a $125-\mathrm{kDa}$ protein encoded by polymerase $\delta$ catalytic subunit gene 1 (POLD1). The p125 catalytic subunit harbors polymerase exonuclease catalytic domain, and serves an important function in cellular growth and differentiation (3). Previous studies suggest that DNA Pol $\delta$ may be involved in tumor progression. Sanefuji et al (4) reported that p125 may serve an important function in tumor invasion, thus leading to a poor prognosis in hepatocellular carcinoma. They found that p125 expression in specimens was significantly correlated with cellular differentiation and the degree of vascular invasion. It was also significantly correlated with abnormal p53 expression, and in vivo studies showed that p125 was upregulated in mutant p53-transfected HepG2 cells. Venkatesan et al (5) mutations at the polymerase active site of DNA Pol $\delta$ may promote genomic instability and accelerate tumorigenesis. A previous study demonstrated that overexpression of POLD1 is associated with an increased risk 
of breast cancer (6). Therefore, DNA pol $\delta$ may a crucial role in tumor progression. However, the expression and clinical value of POLD1 in breast cancer remain unclear.

The aim of the present study was to detect the expression and biologic function of POLD1 in breast cancer, and to investigate its association with clinicopathological characteristics and prognosis in patients with breast cancer.

\section{Materials and methods}

Patients and tissue specimens. A total of 84 female patients with breast cancer were recruited between January 2011 and December 2013 in The Affiliated Tumor Hospital of Guangxi University (Guangxi Zhuang Autonomous Region, China). The mean age of patients was 49 years (range, 34-65 years). The inclusion criteria were as follows: i) Patients with invasive ductal breast carcinoma; ii) patients who underwent mastectomy or wide local excision with axillary surgery; iii) patients with paired fresh carcinoma and adjacent normal tissues; and iv) patients with complete clinical and follow-up information. Exclusion criteria were as follows: i) Patients who received chemotherapy or endocrinotherapy prior to surgery; and ii) lack of pathological data. All patients provided written informed consent, and the study was approved by the Ethics Committee of The Affiliated Tumor Hospital of Guangxi Medical University (Guangxi Zhuang Autonomous Region, China). Fresh tissue specimens were collected immediately after surgical resection. Tumor and adjacent normal $(2 \mathrm{~cm}$ distance from the edge of the tumor) tissues were obtained.

Data collection and follow-up period. Clinical data including age, pathological grade and treatment of patients with breast cancer were collected. Follow-up data were obtained by reviewing the medical records and from direct communication with patients. Following surgery, patients were followed-up until the date of mortality or censored at the date of the last follow-up (March 2016). Disease relapse and metastasis were determined by clinical examination and imaging evaluation, including ultrasonography, X-ray, computed tomography (CT) or magnetic resonance imaging (MRI). The primary endpoint was disease-free survival (DFS), defined as the time interval from surgery to the first evidence of recurrence and metastasis. The follow-up period was 37 months (range, 15-62 months).

Immunohistochemistry. Expression of estrogen receptor (ER), progesterone receptor (PR), human epidermal growth factor receptor 2 (HER-2), p53 and $\mathrm{Ki}-67$ was assessed using immunohistochemistry. A total of 84 samples were subjected to immunohistochemistry. All tissue samples were fixed with $30 \%$ formalin at $28^{\circ} \mathrm{C}$ for $60 \mathrm{~min}$ and then embedded with paraffin. The samples were sectioned to a thickness of 5-mm. The sections were incubated at $60^{\circ} \mathrm{C}$ for $1 \mathrm{~h}$, then placed in xylene I and xylene II for $30 \mathrm{~min}$ in turn. All the slices were dehydrated in a graded ethyl alcohol solution series (100, 95, 90 and $85 \%$ ). Antigen retrieval was performed by placing the slices in $0.01 \mathrm{M}$ potassium citrate solution (Fuzhou Maixin Biotech. Co., Ltd., Fuzhou, China) and microwaved at $90^{\circ} \mathrm{C}$ for $10 \mathrm{~min}$ prior to cooling at room temperature. Slices were then rinsed in PBS three times. Endogenous peroxidase activity was blocked with $3 \% \mathrm{H}_{2} \mathrm{O}_{2}$ and goat serum (Shanghai Haoran
Biological Technology Co., Ltd., Shanghai, China) at $37^{\circ} \mathrm{C}$ for $30 \mathrm{~min}$, then immediately incubated with primary antibody at $4^{\circ} \mathrm{C}$ overnight. The primary antibodies were: ER (dilution, 1:100; catalog no. sc-420; Santa Cruz Biotechnology, Inc., Dallas, TX, USA); PR (dilution, 1:1,000; catalog no. 8753; Cell Signaling Technology, Inc., Danvers, MA, USA); HER-2 (dilution, 1:100; catalogno.sc-33684; SantaCruzBiotechnology, Inc.); p53 (dilution, 1:100; catalog no. sc-47698; Santa Cruz Biotechnology, Inc.); and Ki-67 (dilution, 1:100; catalog no. sc56319; Santa Cruz Biotechnology, Inc.). Subsequently, the slides were washed with PBS three times prior to incubation with corresponding horseradish peroxidase-conjugated mouse anti-goat secondary antibody (dilution, 1:100; catalog no. sc-516246; Santa-Cruz Biotechnology, Inc.) at $37^{\circ} \mathrm{C}$ for $1 \mathrm{~h}$. The slides were then washed three more times in PBS, then stained with DAB (Fuzhou Maixin Biotech. Co., Ltd.) at room temperature for 3-5 min, rinsed 3 times with tap water and stained with $0.5 \%$ hematoxylin (Fuzhou Maixin Biotech. Co., Ltd.), at room temperature for $2 \mathrm{~min}$, prior to sealing using mounting medium (Fuzhou Maixin Biotech. Co., Ltd.). Images were captured using a Leica DMLA light microscope (Leica Microsystems, Inc., Buffalo Grove, IL, USA) with magnifications x200 and x400. Each section was independently evaluated by 2 pathologists, who were blinded to the data of the patients. The intensity was scored as follows: 0 , negative; 1 , weak; 2 , moderate; and 3, strong. The positive area was defined as follows: $0,<5 \% ; 1,5 \%-25 \% ; 2,26 \%-50 \% ; 3,51 \%-75 \%$; and $4,>75 \%$. According to the St Gallen International Expert Consensus (7), the cut-off point for $\mathrm{Ki}-67$ expression was determined as $14 \%,>14 \%$ was considered high expression and $\leq 14 \%$ was considered low expression. p53 expression referred to wild-type p53.

Reverse transcription-quantitative polymerase chain reaction $(R T-q P C R)$. Total RNA was extracted from tissues using TRIzol reagent (Thermo Fisher Scientific, Inc., Waltham, MA, USA) according to the manufacturer's protocol. RNA was reverse-transcribed into cDNA using iScript cDNA Synthesis kit (Bio-Rad Laboratories, Inc., Hercules, CA, USA). qPCR was performed using SYBR Green PCR Master Mix (Qiagen $\mathrm{GmbH}$, Hilden, Germany), according to the manufacturer's protocol. The primer sequences for POLD1 were as follows: 5'-GCTCCGCTCCTACACGCTCAA-3' (forward) and 5'-GGTCTGGTCGTTCCCATTCTGC-3' (reverse). The primer sequences for GAPDH were as follows: 5'-AACGGA TTTGGTCGTATTG-3' (forward) and 5'-CTGGAAGATGGT GATGGG-3' (reverse). The PCR thermocycling conditions were as follows: $95^{\circ} \mathrm{C}$ for $30 \mathrm{sec}$, followed by 40 cycles of $95^{\circ} \mathrm{C}$ for $5 \mathrm{sec}$, and $60^{\circ} \mathrm{C}$ for $30 \mathrm{sec}$. The results were analyzed using the $2^{-\Delta \Delta \mathrm{Cq}}$ method (8) by Bio-Rad CFX Manger 3.0 (Bio-Rad Laboratories, Inc.). Three individual experiments were performed.

Western blot analysis. Paired tumor and adjacent normal tissues were extracted using NP40 lysis buffer (50 mM Tris-Cl, $\mathrm{pH} 7.5,150 \mathrm{mM} \mathrm{NaCl}, 2 \% \mathrm{NP}-40)$. Cellular proteins were extracted using a cell lysis buffer (10 mM Tris-HCl, $\mathrm{pH} 7.4$, $1 \%$ SDS, 1mM Na3VO4). Protein concentration was quantified by bicinchoninic acid protein assay kit. Equal amounts of protein $(20 \mu \mathrm{g})$ were separated by SDS-PAGE (5\% gels) 
and transferred onto polyvinylidene fluoride membranes. Following transfer, membranes were washed with TBS-T solution ( $1 \mathrm{ml}$ TBS solution $+20 \%$ Tween 20$)$, and then blocked with $5 \%$ skimmed milk (in PBS) and agitated at room temperature for $1 \mathrm{~h}$. Next, membranes were incubated at $4^{\circ} \mathrm{C}$ overnight with primary antibodies against p125 (1:1,000; catalog no. 15646-1-AP; ProteinTech Group, Inc., Chicago, IL, USA) and $\beta$-actin (1:1,000; catalog no. sc-130300; Santa Cruz Biotechnology, Inc.). Membranes were washed with $0.01 \%$ Tween-20 (TBST) three times, for 5 min each time, prior to being incubated with horseradish peroxidase-conjugated goat anti-rabbit secondary antibody (1:10,000; catalog no. 7074 ; Cell Signaling Technology, Inc.), agitated at room temperature for $1 \mathrm{~h}$ and washed with $0.01 \%$ Tween-20 (TBST) three times, for $5 \mathrm{~min}$ each time. Immune complexes were detected by incubation with enhanced chemiluminescence western blotting substrate (Thermo Fisher Scientific, Inc., Waltham, MA, USA) and visualized using a LI-COR Odyssey gel imaging scanner (LI-COR Biosciences, Lincoln, NE, USA). Odyssey V3.0 software (LI-COR Biosciences) was used to analyze the image. $\beta$-actin was used as an internal control. Patients with breast cancer were divided into high and low expression group based on the median expression level of p-125.

Cell culture. The human breast adenocarcinoma MCF-7 cell line was obtained from American Type Culture Collection (ATCC; Manassas, VA, USA). The cells were maintained in Dulbecco's modified Eagle's medium (Thermo Fisher Scientific Inc.) supplemented with $10 \%$ fetal bovine serum (Hangzhou Sijiqing Biological Engineering Materials Co., Ltd., Hangzhou, China) at $37^{\circ} \mathrm{C}$ in a humidified atmosphere containing $5 \% \mathrm{CO}_{2}$.

Cell transfection. Short hairpin RNA (shRNA) targeting POLD1 (shPOLD1) and the negative control shRNA (shControl) were purchased from Shanghai GenePharma Co., Ltd. (Shanghai, China). shRNA was designed using online shRNA tools (Invitrogen; Thermo Fisher Scientific, Inc.), and the oligonucleotides encoding POLD1 shRNA were as follows: 5'-CACCGGTCCACCTTCATCCGTATC ACGAATGATACGGATGAAGGTGGACC-3' (forward) and 5'-AAA AGGTCCACCTTCATCCGTATCATTCGT GATACGGATGAAGGTGGACC-3' (reverse). Synthetic interference POLD1 gene sequences were inserted into the shRNA eukaryotic expression vector pLKO. 1-puro plasmid (GenePharma Co., Ltd.). and identified by enzyme digestion and sequencing. For cell transfection, MCF-7 cells $\left(1 \times 10^{5}\right.$ cells/well) were plated in 6-well plates and then transfected with plasmid (shControl and shPOLD1; $500 \mathrm{ng} / \mu \mathrm{l}$ ) for 6 h using Lipofectamine ${ }^{\circledR} 2000$ (Thermo Fisher Scientific, Inc.), according to the manufacturer's protocol. Next, cells were incubated in complete medium (Thermo Fisher Scientific, Inc.) for $48 \mathrm{~h}$, with concentration of $5 \mu \mathrm{g} / \mathrm{ml}$ puromycin screening culture medium for screening of stable transfection cell lines. After $24 \mathrm{~h}$ of infection, the cells were used for subsequent experimentation.

Cell proliferation assay. The proliferation of MCF-7 was assessed using a Cell Counting Kit-8 detection kit (CCK-8; Beyotime Institute of Biotechnology, Haimen, China), as previously described (9). MCF-7 cells were seeded into 96-well plates at a density of $2 \times 10^{3}$ cells/well incubated in 10\% CCK-8 diluted in Dulbecco's modified Eagle's medium at $37^{\circ} \mathrm{C}$. Following transfection, cell proliferation rates were determined at $0,24,48,72$ and $96 \mathrm{~h}$. A microplate reader (set at $450 \mathrm{~nm}$ ) was used to measure the absorbance of each well.

Cell cycle analysis. Flow cytometry was used to determine the cell cycle distribution. Briefly, MCF-7 cells were cultured in 6-well plates and were transfected with shControl or shPOLD1 plasmids. For cell transfection, MCF-7 cells $\left(1 \times 10^{5}\right.$ cells/well) were plated in 6 -well plates and then transfected with plasmid (shControl and shPOLD1; $500 \mathrm{ng} / \mu \mathrm{l}$; Shanghai GenePharma Co., Ltd.) for $6 \mathrm{~h}$ using Lipofectamine 2000 (Thermo Fisher Scientific, Inc.), as aforementioned. After incubation for $96 \mathrm{~h}$, cells were seeded in 6-well culture plates and cultured to $80 \%$ confluence. Cells were harvested and single cell suspensions were prepared in $0.25 \%$ of trypsin (Sigma-Aldrich; Merck KGaA, Darmstadt, Germany). Subsequently, the cells were washed with PBS twice and resuspended at $2 \times 10^{6}$ cells $/ \mathrm{ml}$. Aliquots of $1 \mathrm{ml}$ cells were placed in a $15 \mathrm{ml}$ polypropylene tube on ice and allowed to cool, followed by the addition of $3 \mathrm{ml}$ cold $\left(-20^{\circ} \mathrm{C}\right)$ absolute ethanol. The cells were fixed in $70 \%$ cold ethanol for at least $1 \mathrm{~h}$ at $4^{\circ} \mathrm{C}$. Cells were washed twice with PBS, and $1 \mathrm{ml}$ PI (Sigma-Aldrich; Merck KGaA; $40 \mu \mathrm{g} / \mathrm{ml}$ PI in PBS) staining solution was added to the cell pellet and mixed well. Stained samples were stored for up to a week at $4^{\circ} \mathrm{C}$ in the dark. Analysis of cell cycle was determined on a flow cytometer (BD Biosciences, Franklin Lakes, NJ, USA) and using the CellQuest Pro software (version 5.1; BD Biosciences), as previously described (9).

Statistical analysis. Data were analyzed using SPSS software (version 16.0; SPSS, Inc., Chicago, IL, USA). All results are expressed as the mean \pm standard deviation from three independent replicate experiments. Student's t test was used to analyze the expression of POLD1 level in tumor and adjacent normal specimens. The association between the expression of POLD1 and clinicopathological parameters was assessed using a $\chi^{2}$ test. Survival analysis was performed using Kaplan-Meier method and log-rank tests. Univariate and multivariate Cox regression analyses were used to determine independent prognostic factors. $\mathrm{P}<0.05$ was considered to indicate a statistically significant difference.

\section{Results}

Patient characteristics. A total of 84 females with invasive breast cancer treated with radical mastectomy were retrospectively collected. The diagnosis was confirmed by pathological examination and all patients were diagnosed with duct carcinoma. None of the patients received any therapy prior to surgery. Clinical data including age, tumor size, histological grade, lymph node status and pathological features are presented in Table I. ER, PR, HER-2, p53 status and ki-67 index were routinely evaluated by immunohistochemistry. According to the results, 37 samples were negative for wild-type p53 index, whereas 47 samples demonstrated p53-positive staining (Table I). 
Table I. Clinicopathological characteristics of patients with breast cancer.

\begin{tabular}{|c|c|}
\hline Characteristic & Patients, n (\%) \\
\hline \multicolumn{2}{|l|}{ Age, years } \\
\hline$\leq 45$ & $34(40.5)$ \\
\hline$>45$ & $50(59.5)$ \\
\hline \multicolumn{2}{|l|}{ Tumor size, cm } \\
\hline$\leq 2.5$ & $24(28.6)$ \\
\hline$>2.5$ & $60(71.4)$ \\
\hline \multicolumn{2}{|c|}{ Histological grade } \\
\hline Grade 1 & $18(21.4)$ \\
\hline Grade 2-3 & $66(78.6)$ \\
\hline \multicolumn{2}{|c|}{ Lymph node status } \\
\hline Negative & $31(36.9)$ \\
\hline Positive & $53(63.1)$ \\
\hline \multicolumn{2}{|l|}{ ER status } \\
\hline Negative & $28(33.3)$ \\
\hline Positive & $56(66.7)$ \\
\hline \multicolumn{2}{|l|}{ PR status } \\
\hline Negative & $21(25.0)$ \\
\hline Positive & $63(75.0)$ \\
\hline \multicolumn{2}{|l|}{ HER-2 status } \\
\hline Negative & $40(47.6)$ \\
\hline Positive & $44(52.4)$ \\
\hline \multicolumn{2}{|l|}{ Ki-67 index } \\
\hline$\leq 14 \%$ & $16(19.0)$ \\
\hline$>14 \%$ & $68(81.0)$ \\
\hline \multicolumn{2}{|l|}{ p53 status } \\
\hline Negative & $37(44.0)$ \\
\hline Positive & $47(56.0)$ \\
\hline
\end{tabular}

ER, estrogen receptor; PR, progesterone receptor; HER-2, human epidermal receptor-2.

POLD1 expression in breast cancer. Gene expression level of POLD1 was assessed using RT-qPCR. The results demonstrated that gene expression level of POLD1 was significantly elevated (2.8-fold) in breast cancer tissues compared with that in adjacent normal breast tissues (Fig. 1). Western blot analysis demonstrated that the expression levels of POLD1/p125 subunit were significantly increased (2.2-fold) in cancer tissues compared with adjacent normal breast tissues (Fig. 2).

Association between the expression of POLDI and clinicopathological characteristics. Median expression level of POLD1 was used as the cut-off point (low score, <1.03; high score, $\geq 1.03$ ) and patients with breast cancer were divided into high and low expression groups. The association between the expression of POLD1 and clinicopathological variables in patients with breast cancer was determined. The results demonstrated that increased expression of POLD1 was associated with lymph node metastasis $(\mathrm{P}=0.028)$, histological grade $(\mathrm{P}=0.025)$, p53 status $(\mathrm{P}<0.01)$ and ki-67 index $(\mathrm{P}=0.020$; Table II $)$.
Table II. Association between POLD1 expression and clinicopathological features.

\begin{tabular}{|c|c|c|c|}
\hline \multirow[b]{2}{*}{ Variable } & \multicolumn{2}{|c|}{$\begin{array}{l}\text { POLD1 protein } \\
\text { expression }\end{array}$} & \multirow[b]{2}{*}{ P-value } \\
\hline & Low $(n=41)$ & High $(n=43)$ & \\
\hline Age, years & & & 0.478 \\
\hline$\leq 45$ & 15 & 19 & \\
\hline$>45$ & 26 & 24 & \\
\hline Tumor size, cm & & & 0.073 \\
\hline$\leq 2.5$ & 8 & 16 & \\
\hline$>2.5$ & 33 & 27 & \\
\hline Histological grade & & & 0.025 \\
\hline Grade 1 & 13 & 5 & \\
\hline Grade 2-3 & 28 & 38 & \\
\hline Lymph node status & & & 0.028 \\
\hline Negative & 20 & 11 & \\
\hline Positive & 21 & 32 & \\
\hline ER status & & & 0.123 \\
\hline Negative & 17 & 11 & \\
\hline Positive & 24 & 32 & \\
\hline PR status & & & 0.059 \\
\hline Negative & 14 & 7 & \\
\hline Positive & 27 & 36 & \\
\hline HER-2 status & & & 0.084 \\
\hline Negative & 17 & 23 & \\
\hline Positive & 27 & 17 & \\
\hline $\mathrm{Ki}-67$ index & & & 0.020 \\
\hline$\leq 14 \%$ & 12 & 4 & \\
\hline$>14 \%$ & 29 & 39 & \\
\hline p53 status & & & $<0.001$ \\
\hline Negative & 9 & 28 & \\
\hline Positive & 32 & 15 & \\
\hline
\end{tabular}

ER, estrogen receptor; PR, progesterone receptor; HER-2, human epidermal receptor-2; POLD1, polymerase $\delta$ catalytic subunit gene.

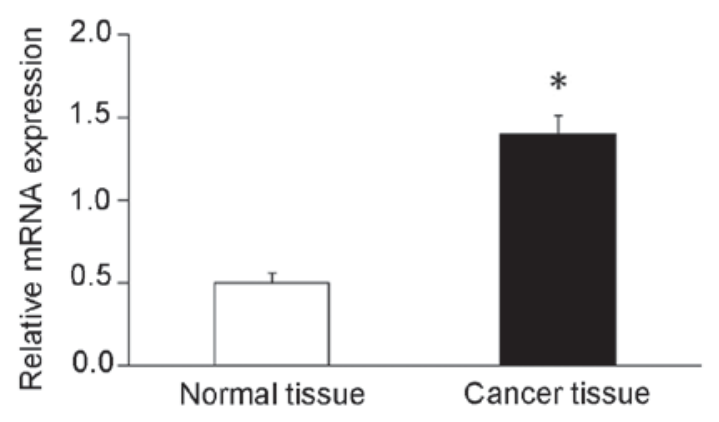

Figure 1. Relative expression of POLD1 mRNA in breast cancer and adjacent normal tissues. ${ }^{*} \mathrm{P}<0.05$. POLD1, polymerase $\delta$ catalytic subunit gene 1 .

However, the expression of POLD1 was not associated with the age, tumor size, ER, PR and HER-2 status (P>0.05; Table II). 
A

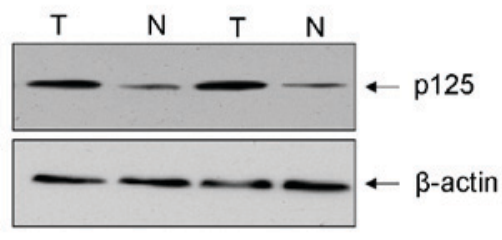

B

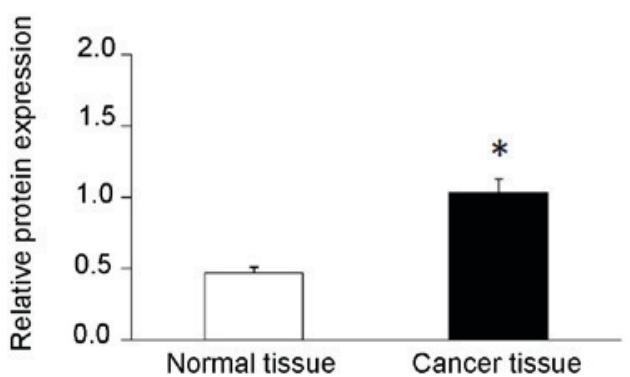

Figure 2. Expression of POLD1 protein (p125) in breast cancer and adjacent normal tissues. (A) Western blot analysis of protein expression levels of p125. $\beta$-actin was used as an endogenous control. (B) Relative expression levels of p125 in breast cancer tissues and adjacent normal tissues. "P<0.05. T, tumor tissues; $\mathrm{N}$, adjacent normal tissues; POLD1, polymerase $\delta$ catalytic subunit gene 1 .

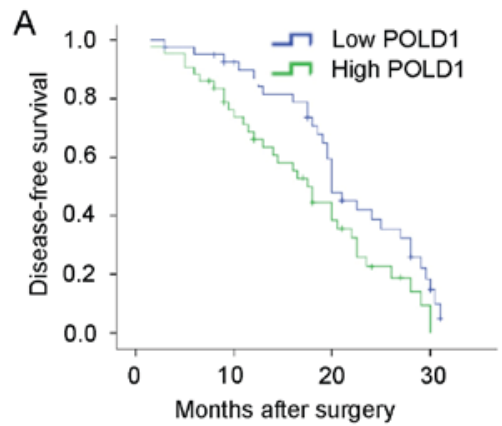

C

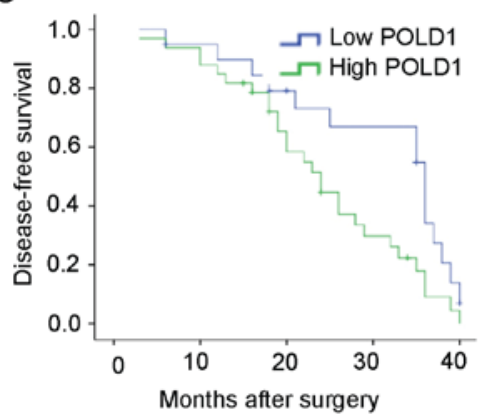

B

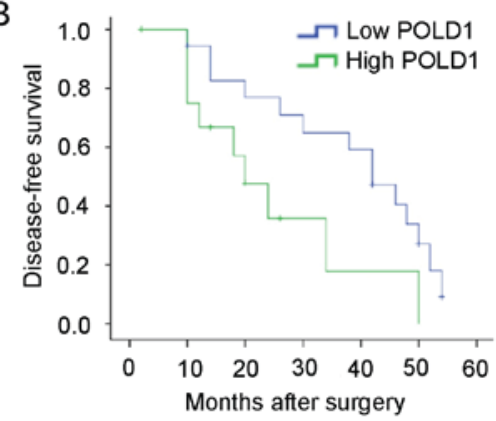

D

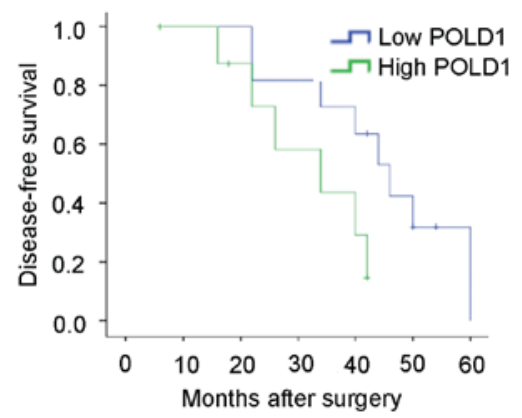

Figure 3. Kaplan-Meier curves for disease-free survival rates of 84 patients with breast cancer. (A) Disease-free survival rates of patients with high ( $\mathrm{n}=43$ ) and low ( $\mathrm{n}=41$ ) expression of POLD1. $\mathrm{P}=0.033$. (B) High expression of POLD1 was associated with poor disease-free survival at early-stage ( $=31$ ). $\mathrm{P}=0.037$. (C) High expression of POLD1 was associated with poor DFS at late-stage ( $=53)$. $P=0.023$. (D) High expression of POLD1 was associated with poor POLD1 in patients with triple-negative tumors $(n=20)$. $P=0.049$. POLD1, polymerase $\delta$ catalytic subunit gene 1 .

Prognostic value of POLDl expression in patients with breast cancer. To further determine the importance of increased expression of POLD1 in breast cancer, DFS was assessed in patients with breast cancer patients using Kaplan-Meier method and log-rank tests. The results indicated that patients with increased expression of POLD1 exhibited shorter DFS compared with patients with low expression of POLD1 (median, 31.5 vs. 38.6 months; $\mathrm{P}=0.033$; Fig. $3 \mathrm{~A}$ ). Additionally, patients with increased expression of POLD1 exhibited shorter DFS compared with those in the low expression group POLD1 at early stage $(\mathrm{P}=0.037)$ or late stage $(\mathrm{P}=0.023)$ (Fig. 3B and $\mathrm{C})$. Increased expression of POLD1 was associated with shorter DFS in patients with triple-negative tumors ( $\mathrm{P}=0.049$; Fig. 3D).

Univariate analysis demonstrated that histological grade $(\mathrm{P}=0.018)$, lymph node status $(\mathrm{P}=0.001), \mathrm{ki}-67$ index $(\mathrm{P}=0.019)$ and POLD1 expression status $(\mathrm{P}<0.01)$ were associated with DFS. These results suggest that POLD1 may be a valuable prognostic factor in breast cancer. Multivariate analysis revealed that expression of POLD1 [Hazard ratio (HR), 2.14; $\mathrm{P}=0.048$ ], histological grade (HR, 4.46; $\mathrm{P}=0.010)$, lymph node status (HR, 2.13; $\mathrm{P}=0.045)$ and ki-67 expression $(\mathrm{HR}, 4.61 ; \mathrm{P}=0.021)$ were independent factors associated with DFS (Table III).

shPOLD1 inhibits the proliferation of MCF7 cells. To investigate the function of POLD1 in cellular proliferation in breast cancer, MCF-7 cells were transfected with shPOLD1 and cell proliferation was assessed using a CCK-8 assay. The expression of POLD1 was significantly downregulated following transfection with shPOLD1 (Fig. 4A). Downregulation of POLD1 significantly inhibited cell proliferation compared with the control $(\mathrm{P}<0.01$; Fig. 4B).

shPOLD suppresses cell cycle progression. Following transient transfection of MCF-7 cells with shPOLD1 or shControl, 
Table III. Univariate and multivariate analysis of clinicopathological factors for the DFS of 84 patients with breast cancer.

Univariate analysis

Multivariate analysis

\begin{tabular}{lcccr}
\cline { 2 - 3 } Characteristic & HR $(95 \% \mathrm{CI})$ & P-value & HR $(95 \% \mathrm{CI})$ & P-value \\
\hline Age & $0.81(0.45-1.48)$ & 0.387 & & \\
Tumor size & $1.61(0.88-2.93)$ & 0.119 & & \\
Histological grade & $2.99(1.20-7.45)$ & 0.018 & & \\
Lymph node status & $2.85(1.50-5.38)$ & 0.001 & $2.13(1.07-13.94)$ & 0.010 \\
ER status & $0.73(0.36-1.47)$ & 0.376 & & \\
HER-2 status & $1.17(0.59-2.32)$ & 0.654 & & 0.021 \\
Ki-67 & $3.52(1.23-10.12)$ & 0.019 & $4.61(1.26-16.95)$ & 0.048 \\
POLD1 & $3.14(1.67-5.91)$ & $<0.001$ & $2.14(0.99-4.68)$ &
\end{tabular}

DFS, disease-free survival; ER, estrogen receptor; HER-2, human epidermal receptor-2; POLD1, polymerase $\delta$ catalytic subunit gene.

A

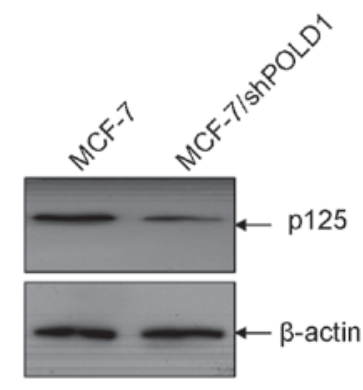

B

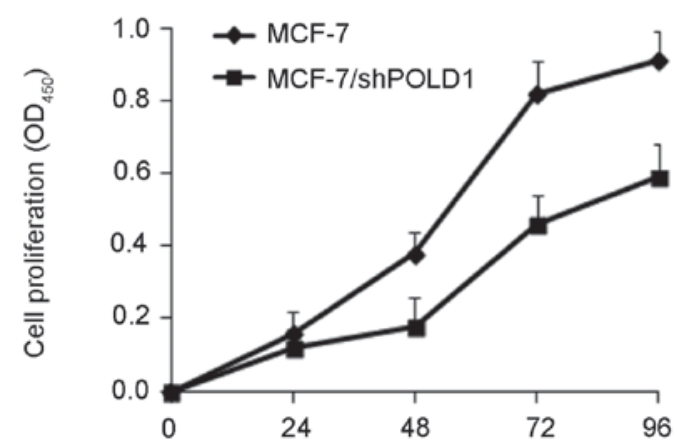

Figure 4. shPOLD1 inhibits the proliferation of MCF-7 cells. (A) The expression of POLD1 was significantly downregulated following transfection with shPOLD1. (B) POLD1 shRNA inhibits the proliferation of MCF7 cells. POLD1, polymerase $\delta$ catalytic subunit gene 1; shPOLD1, short hairpin RNA targeting POLD1.

cell cycle distribution was analyzed using flow cytometry. The results demonstrated that the percentage of cells in $G_{1}$ phase was increased following transfection with shPOLD1 compared with that in the control $(\mathrm{P}<0.05$; Fig. 5), thereby suggesting that POLD1 shRNA may inhibit the cell cycle at $\mathrm{G}_{1}$ phase in MCF-7 cells.

\section{Discussion}

POLD1 belongs to a family of human DNA polymerases and exhibits polymerase and 3 ' to 5'exonuclease activity. POLD1 is responsible for the synthesis of the lagging strand during DNA replication (10). It was recently demonstrated that POLD1 may modulate cell cycle progression and promote the proliferation of cancer cells (11). A previous study revealed that the expression of POLD1 was increased in human breast cancer cells (9). To the best of our knowledge, the present study is the first to explore the association of the expression of POLD1 with clinicopathological variables and survival time in patients with breast cancer.

Breast cancer is a common cancer in females. In the USA, $>230,000$ new cases of breast cancer were diagnosed and >40,000 mortalities were reported in 2015 (12). Despite advances, the clinical prognosis of breast cancer remains poor. Identification of novel and reliable prognostic factors is required for the development of therapeutic strategies in breast cancer.

Abnormal expression of POLD1 has been detected in several types of solid tumors. However, the expression and clinical value of POLD1 in breast cancer remains unclear (3). The results of the present study demonstrated that gene and protein expression of POLD1 was elevated in breast cancer tissues compared with adjacent normal tissues. Additionally, increased expression of POLD1 was associated with lymph node metastasis, differentiation grade, p53 status and ki-67 index, whereas there was no association with the remaining clinicopathological variables. Additionally, the association between POLD1 and breast cancer prognosis was assessed through survival analysis. The results demonstrated that increased expression of POLD1 was associated with shorter DFS in patients with breast cancer. Multivariate analysis revealed that the expression of POLD1, together with axillary lymph node status, histological grade and ki-67 index may be considered as independent prognostic factors for long-term outcomes in patients with breast cancer. Therefore, POLD1 

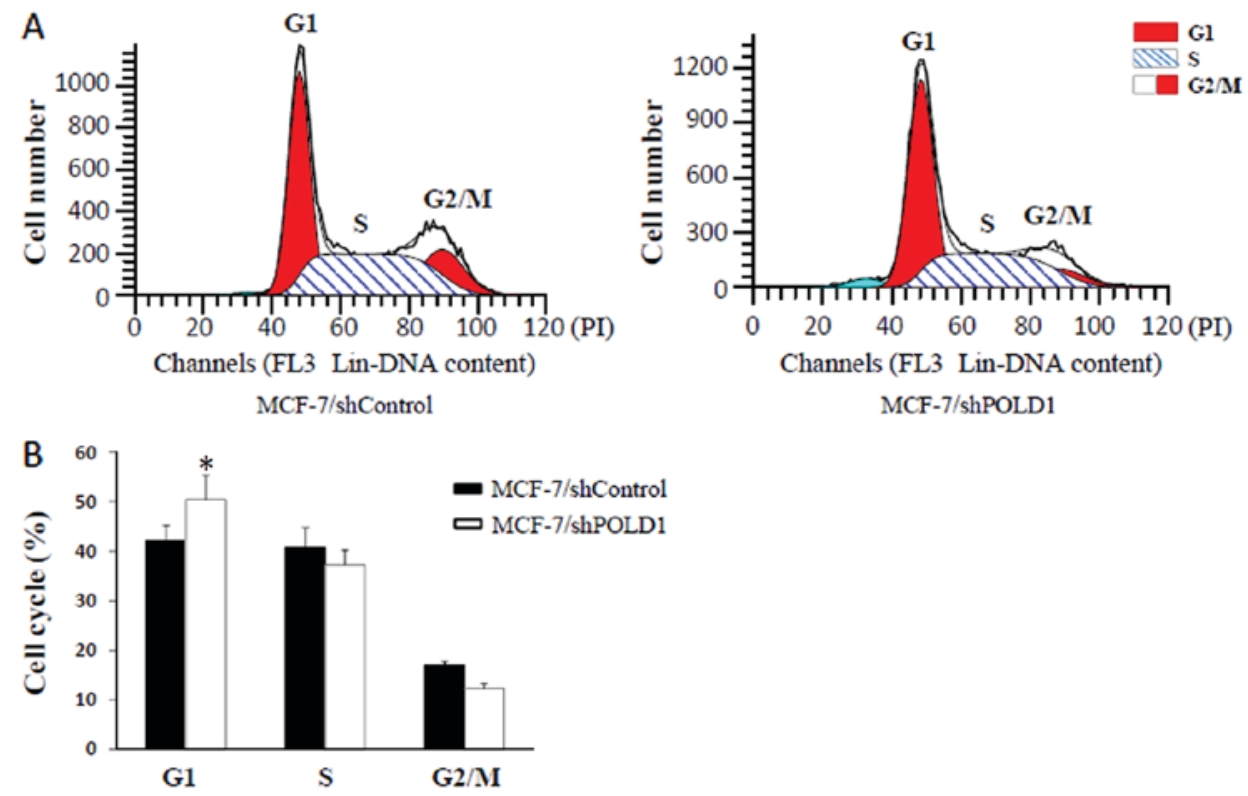

Figure 5. POLD1 knockdown affects cell cycle progression. (A) Cell cycle distribution of the MCF-7/shControl and MCF-7/shPOLD1 cells was analyzed by flow cytometry. (B) Data for the cell cycle assay results. Data points are presented as the mean \pm standard error of the mean. " $\mathrm{P}<0.01$, shControl vs. shPOLD1. POLD1, polymerase $\delta$ catalytic subunit gene 1 ; shPOLD1, short hairpin RNA targeting POLD1; shControl, control short hairpin RNA.

is a potential prognostic factor and therapeutic target in patients with invasive breast carcinoma.

The results of the present study are consistent with those of a previous study which reported that the expression of POLD1 is associated with cellular differentiation and the degree of vascular invasion in hepatocellular carcinoma (4), thus leading to poor prognosis. A previous study identified that POLD1 may be used as a novel biomarker for head and neck cancer (13). Narayan et al (14) employed cDNA array comparative genomic hybridization to analyze 29 cervical cancer cases and detected increased expression of POLD1 in the tissues. Previous studies have demonstrated that genetic mutations in the POLD1 gene are associated with epithelial (15), endometrium (16) and colorectal cancer (17). The hypothesis of the present study was that POLD1 may be associated with tumorigenesis and tumor progression, and may regulate proliferation and cell cycle progression. A previous study reported that inhibition of POLD1 promoted apoptosis of hepatocellular carcinoma cells (18). Additionally, POLD1 may serve an important function in cell cycle progression and repair of damaged DNA (19). POLD1 may be overexpressed in mesothelioma and its expression is associated with pemetrexed/carboplatin resistance (20), thus suggesting that POLD1 may provide protection against DNA damage through multiple molecular mechanisms.

p53 may inhibit the expression of p125 by regulating the methylation of POLD1 gene promoter (9). POLD1 is a transcriptional target of p53 (21), therefore the association between the expression of wild-type p53 and POLD1 in breast cancer was assessed in the present study. The results demonstrated that the expression of POLD1was negatively associated with the expression of wild-type p53. Previous studies demonstrated that POLD1 may be upregulated in mutant p53-transfected cells (4). Further studies investigating the association between the expression of mutant-type p53 and POLD1 in clinical samples are required.
TNBC is a subtype of breast cancer, defined by lack of expression of ER, PR and HER-2, and is associated with poor prognosis (22). Further studies identifying biomarkers in TNBC are required. The results of the present study demonstrated that the expression of POLD1 was not associated with ER, PR, HER-2, thus suggesting that there is no association between the expression of POLD1 and TNBC. However, POLD1 was associated with prognostic factors, including lymph node metastasis and histological grade. Survival analysis indicated that the expression of POLD1 was associated with shorter DFS in patients with TNBC. The results suggested that the prognosis of breast cancer is not associated with molecular subtypes and that a more reliable prognostic factor is required. A previous study demonstrated that POLD1 downregulation by shRNA suppressed cell proliferation, cell cycle progression and DNA synthesis in HEK293 cells (19), suggested that POLD1 plays important role in the regulation of cell cycle progression; therefore, the effects of POLD1 in regulating cell cycle and proliferation of breast cancer cells was examined in MCF-7 cells in vitro. The results revealed that downregulation of POLD1 suppressed cell cycle progression and cell proliferation in MCF-7 cells, thus suggesting that POLD1 may serve an important function in tumor progression.

The present study has several limitations. In the present study the proportion of HER-2 positive cells is high compared with previous studies (23). This may be due to the small sample size. Although protein expression of POLD1 was detected using western blot analysis, its expression should be also validated using immunohistochemistry. Additionally, further studies are required to investigate the molecular mechanisms underlying the effects of POLD1 in breast cancer.

In conclusion, POLD1 may serve an important function in breast cancer progression, and may be considered as a novel therapeutic target in breast cancer. 


\section{Acknowledgements}

Not applicable.

\section{Funding}

The present study was supported by National Natural Science Foundation of China (grant no. 81360396) and Natural Science Foundation of Guangxi (grant no. 2015GXNSFAA139204).

\section{Availability of data and materials}

The datasets used and/or analyzed during the current study are available from the corresponding author on reasonable request.

\section{Authors' contributions}

CW, QQT and QT conceived and designed the study, performed patient collection and clinical data interpretation, and wrote the manuscript. JL and WY helped with the tissue preparation and conducted the in vitro experiments. QM and BL performed the statistical analysis. All authors read and approved the final manuscript.

\section{Ethics approval and consent to participate}

The present study was approved by the Ethics Committee of The Affiliated Tumor Hospital of Guangxi Medical University (Guangxi Zhuang, China) and all participants provided written informed consent.

\section{Patient consent for publication}

Written informed consent was obtained from all participants for the publication of their data.

\section{Competing interests}

The authors declare that they have no competing interests.

\section{References}

1. Torre LA, Bray F, Siegel RL, Ferlay J, Lortet-Tieulent J and Jemal A: Global cancer statistics, 2012. CA Cancer J Clin 65 87-108, 2015

2. Liu L, Mo J, Rodriguez-Belmonte EM and Lee MY: Identification of a fourth subunit of mammalian DNA polymerase delta. J Biol Chem 275: 18739-18744, 2000.

3. Nicolas E, Golemis EA and Arora S: POLD1: Central mediator of DNA replication and repair, and implication in cancer and other pathologies. Gene 590: 128-141, 2016.

4. Sanefuji K, Taketomi A, Iguchi T, Sugimachi K, Ikegami T, Yamashita Y, Gion T, Soejima Y, Shirabe K and Maehara Y: Significance of DNA polymerase delta catalytic subunit p125 induced by mutant p53 in the invasive potential of human hepatocellular carcinoma. Oncology 79: 229-237, 2010.

5. Venkatesan RN, Treuting PM, Fuller ED, Goldsby RE, Norwood TH, Gooley TA, Ladiges WC, Preston BD and Loeb LA: Mutation at the polymerase active site of mouse DNA polymerase delta increases genomic instability and accelerates tumorigenesis. Mol Cell Biol 27: 7669-7682, 2007.

6. Sigurdson AJ, Hauptmann M, Chatterjee N, Alexander BH, Doody MM, Rutter JL and Struewing JP: Kin-cohort estimates for familial breast cancer risk in relation to variants in DNA base excision repair, BRCA1 interacting and growth factor genes. BMC Cancer 4: 9, 2004.
7. Goldhirsch A, Wood WC, Coates AS, Gelber RD, Thurlimann B and Senn HJ; Panel members: Strategies for subtypes-dealing with the diversity of breast cancer: Highlights of the St. Gallen international expert consensus on the primary therapy of early breast Cancer 2011. Ann Oncol 22: 1736-1747, 2011.

8. Livak KJ and Schmittgen TD: Analysis of relative gene expression data using real-time quantitative PCR and the 2(-Delta Delta C(T)) method. Methods 25: 402-408, 2001

9. Zhang L, Yang W, Zhu X and Wei C: p53 inhibits the expression of p125 and the methylation of POLD1 gene promoter by downregulating the Sp1-induced DNMT1 activities in breast cancer. Onco Targets Ther 9: 1351-1360, 2016.

10. Lessel D, Hisama FM, Szakszon K, Saha B, Sanjuanelo AB, Salbert BA, Steele PD, Baldwin J, Brown WT, Piussan C, et al: POLD1 Germline mutations in patients initially diagnosed with Werner syndrome. Hum Mutat 36: 1070-1079, 2015.

11. Kashkin K, Chernov I, Stukacheva E, Monastyrskaya G, Uspenskaya N, Kopantzev E and Sverdlov E: Cancer specificity of promoters of the genes controlling cell proliferation. J Cell Biochem 116: 299-309, 2015.

12. Siegel RL, Miller KD and Jemal A: Cancer statistics, 2015. CA Cancer J Clin 65: 5-29, 2015.

13. Ceder R, Haig Y, Merne M, Hansson A, Zheng X, Roberg K, Nees M, Iljin K, Bloor BK, Morgan PR, et al: Differentiation-promoting culture of competent and noncompetent keratinocytes identifies biomarkers for head and neck cancer. Am J Pathol 180: 457-472, 2012.

14. Narayan G, Bourdon V, Chaganti S, Arias-Pulido H, Nandula SV, Rao PH, Gissmann L, Dürst M, Schneider A, Pothuri B, et al: Gene dosage alterations revealed by cDNA microarray analysis in cervical cancer: Identification of candidate amplified and overexpressed genes. Genes Chromosomes Cancer 46: 373-384, 2007.

15. Goldsby RE, Hays LE, Chen X, Olmsted EA, Slayton WB Spangrude GJ and Preston BD: High incidence of epithelial cancers in mice deficient for DNA polymerase delta proofreading. Proc Natl Acad Sci USA 99: 15560-15565, 2002.

16. Wong A, Kuick CH, Wong WL, Tham JM, Mansor S, Loh E, Jain S, Vikas NN, Tan SH, Chan SH, et al: Mutation spectrum of POLE and POLD1 mutations in South East Asian women presenting with grade 3 endometrioid endometrial carcinomas. Gynecol Oncol 141: 113-120, 2016.

17. Palles C, Cazier JB, Howarth KM, Domingo E, Jones AM, Broderick P, Kemp Z, Spain SL, Guarino E, Salguero I, et al: Germline mutations affecting the proofreading domains of POLE and POLD1 predispose to colorectal adenomas and carcinomas. Nat Genet 45: 136-144, 2013.

18. Cao B, Zhang Z, Zhang Y, Li J, Liang G and Ling J: Effect of Smilax china L.-containing serum on the expression of POLD1 mRNA in human hepatocarcinoma SMMC-7721 cells. Exp Ther Med 6: 1070-1076, 2013.

19. Song J, Hong P, Liu C, Zhang Y, Wang J and Wang P: Human POLD1 modulates cell cycle progression and DNA damage repair. BMC Biochem 16: 14, 2015.

20. Roe OD, Szulkin A, Anderssen E, Flatberg A, Sandeck H, Amundsen T, Erlandsen SE, Dobra K and Sundstrøm SH: Molecular resistance fingerprint of pemetrexed and platinum in a long-term survivor of mesothelioma. PLoS One 7: e40521, 2012.

21. Li B and Lee MY: Transcriptional regulation of the human DNA polymerase delta catalytic subunit gene POLD1 by p53 tumor suppressor and Sp1. J Biol Chem 276: 29729-29739, 2001.

22. Lee A and Djamgoz MBA: Triple negative breast cancer: Emerging therapeutic modalities and novel combination therapies. Cancer Treat Rev 62: 110-122, 2018.

23. Chang HR: Trastuzumab-based neoadjuvant therapy in patients with HER2-positive breast cancer. Cancer 116: 2856-2867, 2010 .

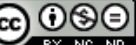

This work is licensed under a Creative Commons Attribution-NonCommercial-NoDerivatives 4.0 International (CC BY-NC-ND 4.0) License. 